\title{
Reversing-Pulse Electric Birefringence of Poly $(\gamma$-benzyl L-glutamate) $V$. Field-Induced Changes of Electric and Hydrodynamic Properties in Helix-Forming Solvents as Revealed by Transient Signals
}

\author{
Kiwamu Yamaoka,* Shinobu Yamamoto, Manabu Kimura, \\ and Isao KosaKo \\ Faculty of Science, Hiroshima University, Higashisenda-machi, \\ Naka-ku, Hiroshima 730, Japan
}

(Received October 19, 1990)

\begin{abstract}
Reversing-pulse electric birefringence (RPEB) of poly( $\gamma$-benzyl L-glutamate), [Glu $(\mathrm{OBzl})]_{n}$, with a weight-average molecular weight of $1.71 \times 10^{5}$ was measured at $20^{\circ} \mathrm{C}$ and at $535 \mathrm{~nm}$ in helix-forming organic solvents in the low field strength region $\left(0-5 \mathrm{k} \mathrm{V} \mathrm{cm}^{-1}\right)$, with an emphasis on the transient signal. The theoretical formalism was given for detecting the effect of an applied electric pulse field on reversible change in the electric and hydrodynamic properties of $[\mathrm{Glu}(\mathrm{OBzl})]_{n}$ from the normalized signal profiles, areas, and initial slopes of an RPEB signal. A standard experimental procedure was given for unraveling the field-induced change of the electric and hydrodynamic parameters, by utilizing the areas of buildup $\left(A_{\mathrm{B}}\right)$, reverse $\left(A_{\mathrm{R}}\right)$, and decay $\left(A_{\mathrm{D}}\right)$ processes at the limiting low field. The area ratio, $A_{\mathrm{B}} /\left[(1 / 2) A_{\mathrm{R}}+A_{\mathrm{D}}\right]=1$, should hold regardless of the degree of polydispersity of a sample, if no direct field effect on polymer conformation is present. The ratio was found to be 0.95 in cyclohexanone, 0.93 in 2 -chloroethanol, 0.81 in pyridine, and $0.74-0.81$ in chloroform, all being less than unity. Thus, the electric moments and/or the chain length of the $[\mathrm{Glu}(\mathrm{OBzl})]_{n}$ helix were verified to change reversibly to varying extent during the electric field orientation.

KEY WORDS Reversing-Pulse Electric Birefringence / Poly( $\gamma$-benzyl Lglutamate) / Field Effect / Electro-Hydrodynamic Property / Area Method /
\end{abstract}

With the aim of perfecting the reversingpulse electric birefringence (RPEB) method and applying it to macromolecules in solution to elucidate the electric, optical and hydrodynamic properties, the RPEB study has been carried out in our laboratory on many biopolymers and related polyelectrolytes. ${ }^{1-16}$ A major problem associated with these systems is polydispersity regarding the molecular weight and chain length. This problem is almost always encountered with natural macromolecules of biological interest, which are prepared by extraction from living organisms, and also with the synthetic polymers, which are prepared by in vitro polymerization. Thus, the polydispersity effect must be taken into account for quantitative analysis of the electric birefringence signal. ${ }^{17}$ Yoshioka and his coworkers were the first who seriously considered this effect in the analysis of the single square-wave pulse electric birefringence (SPEB) data of poly ( $\alpha$-L-glutamic acid). ${ }^{18-21}$ The RPEB method is usually superior to the conventional SPEB method in that the transient signal at low fields can yield sufficient information on the polydispersity effect and the electric and hydrodynamic properties of the rodlike or helical polymers. ${ }^{17}$

\footnotetext{
* To whom correspondence should be addressed.
} 
Another major problem associated with an orienting electric pulse field is the direct field effect on the polymer conformation, which may change concurrently with molecular orientation. This field effect has seldom been studied mostly because of the difficulty involved in detecting such field-induced conformational changes for polydisperse samples. Unless anomalies are observed in the birefringence signal, which usually appears normal in the low field strength region, the steady-state data are interpreted in a straightforward manner with the aid of theories for rodlike polymers. ${ }^{17,22}$ Such anomalous signals have occasionally been reported to appear at very high fields and attributed to field-induced helix-coil or helixhelix transitions. ${ }^{23-29}$ Only recently, quantitative analysis of RPEB measurements has been advanced to detect the field-on structural changes at weak fields, even if the signal pattern reveals no anomaly; for example, the effect of applied fields on aggregation ${ }^{1}$ or the length of helix ${ }^{11}$ has been clarified.

In a previous paper of this series, ${ }^{15}$ the RPEB study of the electrooptical and hydrodynamic properties of poly $(\gamma$-benzyl L-glutamate), $[\mathrm{Glu}(\mathrm{OBzl})]_{n}$, was reported with a particular emphasis on the field-strength dependence of the field-on (the reverse) and field-off (the decay) processes. The present work is an extension of the previous study to the transient RPEB behavior of a fractionated and slightly polydisperse $[\mathrm{Glu}(\mathrm{OBzl})]_{n}$ sample in the helix-forming solvents at the low-filed (i.e., the Kerr-law) region, in order to detect the possible field effect on polymer structures. For this purpose, attention is drawn to the characteristics of the transient RPEB signal patterns of the buildup, reverse, and decay processes, since such quantities as areas and initial slopes may now be available with sophisticated apparatus. The experimental results obtained in the low field region will be analyzed with a newly devised procedure, the area method. ${ }^{13}$

\section{THEORETICAL}

Suppose that a polydisperse polymer system consists of nonconducting, cylindrically symmetric molecules in dilute solution, whose lengths, $l$, are distributed continuously over the $[0, \infty]$ range, and that each molecule possesses the permanent electric dipole moment along the symmetric axis $\mu(l)$ and the polarizability anisotropy $\Delta \alpha(l)\left(=\alpha_{33}-\alpha_{11}\right) .^{5,11,17}$ The characteristics of an RPEB signal are schematically shown in Figure $19^{9,11-15}$ In addition to the normalized signal profiles $\left(\Delta_{\mathrm{B}}(t)\right.$ for buildup, $\Delta_{\mathbf{R}}(t)$ for reverse, and $\Delta_{\mathrm{D}}(t)$ for decay) at a given time $t$ with thick lines, the corresponding areas, represented with three shaded portions $\left(A_{\mathrm{B}}, A_{\mathrm{R}}\right.$, and $A_{\mathrm{D}}$ ), and initial slopes with arrows at $t=0$ $\left(S_{\mathrm{B}}, S_{\mathrm{R}}\right.$, and $\left.S_{\mathrm{D}}\right)$ may be determined experimentally. These quantities can also be calculated theoretically for the polydisperse polymer system. The theoretical expressions for the reversing birefringence signal, $\Delta n(t)=$ $n_{\|}(t)-n_{\perp}(t)$, were originally derived only for the limiting low field, ${ }^{17}$ and later extended. ${ }^{30}$ More general expressions were recently derived for higher fields, ${ }^{31,32}$ but they may be given only by complex mult-termed functions. In APPENDIX, the theoretical expressions are

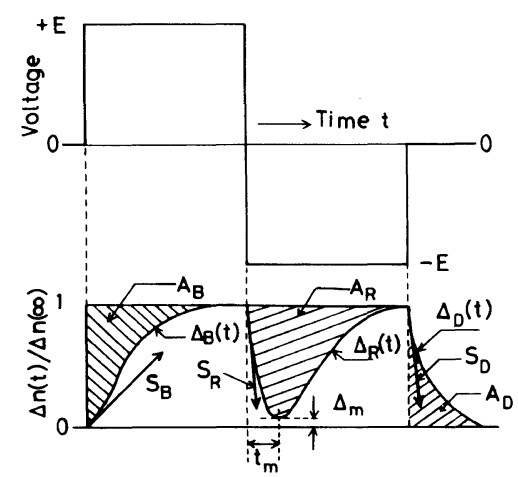

Figure 1. Schematic presentation of an applied electric reversing-pulse (upper) and a normalized RPEB signal (lower). Transient profiles by thick lines: $\Delta_{\mathrm{B}}(t), \Delta_{\mathrm{R}}(t)$, and $\Delta_{\mathrm{D}}(t)$. Areas by slanted lines: $A_{\mathrm{B}}, A_{\mathrm{R}}$, and $A_{\mathrm{D}}$. Initial slopes by arrows: $S_{\mathrm{B}}, S_{\mathrm{R}}$, and $S_{\mathrm{D}}$. Subscripts $\mathrm{B}, \mathrm{R}$, and D stand for the buildup, reverse, and decay processes. $t_{\mathrm{m}}$, time required for the extremum $\Delta_{\mathrm{m}}$ in the reverse portion. 
summarized for the buildup, reverse, and decay processes.

Relations between Profiles, Areas, and Initial Slopes

If both polymer conformation and electrooptic properties remain unaltered by an applied electric field, there exist three simple but useful relationships in the transient processes of a given RPEB signal in the low field region where the Kerr law is obeyed. From eq A1, A4, and A7, the normalized birefringence profiles at a given time $t$ are related to each other as ${ }^{13,30}$

$$
\Delta_{\mathrm{B}}(t)=\frac{1}{2}\left(1+\Delta_{\mathrm{R}}(t)\right)-\Delta_{\mathrm{D}}(t)
$$

From eq A2, A5, and A8, the following expression results for the areas:

$$
A_{\mathrm{B}}=\frac{1}{2} A_{\mathrm{R}}+A_{\mathrm{D}}
$$

This formula indicates that the area ratio, defined as $A_{\mathrm{B}} /\left[(1 / 2) A_{\mathrm{R}}+A_{\mathrm{D}}\right]$, is unity regardless of the degree of polydispersity for a given polymer sample. ${ }^{13}$ Finally, from eq A3, A6, and A9, the following relationship holds for the initial slopes:

$$
S_{\mathrm{B}}=\frac{1}{2} S_{\mathrm{R}}-S_{\mathrm{D}}
$$

Thus, eq $1-3$ are the so-called indicators for detecting any field-induced transitions of a rodlike polymer system.

\section{Differently-Averaged Relaxation Times and}

\section{Electric Properties}

The permanent electric dipole moment and the covalent (electronic and atomic) polarizability anisotropy for a long cylindrical molecule can be assumed to be proportional to the length, i.e., $\mu(l) \propto l$ and $\Delta \alpha(l) \propto l l^{5}$ The fraction of molecules with length $l, \phi(l)$, may be assumed to be proportional to the $l f_{n}(l)$, where $f_{n}(l)$ is the continuous probability distribution function of $l$ based on the number of solute in solution. ${ }^{5,13,33}$ Thus, the following two important expressions result for such a polydisperse system:

$$
\begin{array}{r}
\left\langle\tau \beta^{2}\right\rangle_{w}=\frac{\int_{0}^{\infty} \tau(l) \beta(l)^{2} l f_{n}(l) \mathrm{d} l}{\int_{0}^{\infty} l f_{n}(l) \mathrm{d} l}=\langle\tau\rangle_{z+1}\left\langle\beta^{2}\right\rangle_{w} \\
\langle\tau \gamma\rangle_{w}=\frac{\int_{0}^{\infty} \tau(l) \gamma(l) l f_{n}(l) \mathrm{d} l}{\int_{0}^{\infty} l f_{n}(l) \mathrm{d} l}=\langle\tau\rangle_{z}\langle\gamma\rangle_{w}
\end{array}
$$

The weight-average mean-square permanent dipole moment $\left\langle\mu^{2}\right\rangle_{w}$ equals the product of the $z$ - and weight-averages. ${ }^{14}$ By combination of eq 4 and 5 with eq A2, A5, A8, and A10, the areas in the Kerr-law region may be expressed as follows:

$$
\begin{aligned}
A_{\mathrm{B}} & =\frac{4\left\langle r_{\mathrm{B}}\right\rangle_{w}}{\left\langle r_{\mathrm{B}}\right\rangle_{w}+1} \cdot\left\langle\tau_{\mathrm{B}}\right\rangle_{z+1}+\frac{1}{\left\langle r_{\mathrm{B}}\right\rangle_{w}+1} \cdot\left\langle\tau_{\mathrm{B}}\right\rangle_{z}(6) \\
A_{\mathrm{R}} & =\frac{6\left\langle r_{\mathrm{R}}\right\rangle_{w}}{\left\langle r_{\mathrm{R}}\right\rangle_{w}+1} \cdot\left\langle\tau_{\mathrm{R}}\right\rangle_{z+1} \\
A_{\mathrm{D}} & =\frac{\left\langle r_{\mathrm{D}}\right\rangle_{w}}{\left\langle r_{\mathrm{D}}\right\rangle_{w}+1} \cdot\left\langle\tau_{\mathrm{D}}\right\rangle_{z+1}+\frac{1}{\left\langle r_{\mathrm{D}}\right\rangle_{w}+1} \cdot\left\langle\tau_{\mathrm{D}}\right\rangle_{z}(8)
\end{aligned}
$$

where $\langle\tau\rangle_{z+1}$ and $\langle\tau\rangle_{z}$ are the $(z+1)$ and $z$-average rotational relaxation times, respectively, and $\langle r\rangle_{w} \equiv\left\langle\beta^{2}\right\rangle_{w} / 2\langle\gamma\rangle_{w}=\left\langle\mu^{2}\right\rangle_{w} /$ $\langle\Delta \alpha\rangle_{w} k T$ ( $k$ is the Boltzmann constant and $T$ is the absolute temperature). Similarly, from eq A3, A6, A9, and A10, the initial slopes are given as

$$
\begin{aligned}
& S_{\mathrm{B}}=\frac{1}{\left\langle r_{\mathrm{B}}\right\rangle_{w}+1} \cdot\left\langle\frac{1}{\tau_{\mathrm{B}}}\right\rangle_{z} \\
& S_{\mathrm{R}}=-\frac{2\left\langle r_{\mathrm{R}}\right\rangle_{w}}{\left\langle r_{\mathrm{R}}\right\rangle_{w}+1} \cdot\left\langle\frac{1}{\tau_{\mathrm{R}}}\right\rangle_{z+1} \\
& S_{\mathrm{D}}=-\frac{\left\langle r_{\mathrm{D}}\right\rangle_{w}}{\left\langle r_{\mathrm{D}}\right\rangle_{w}-1} \cdot\left\langle\frac{1}{\tau_{\mathrm{D}}}\right\rangle_{z+1}
\end{aligned}
$$




$$
-\frac{1}{\left\langle r_{\mathrm{D}}\right\rangle_{w}+1} \cdot\left\langle\frac{1}{\tau_{\mathrm{D}}}\right\rangle_{z}
$$

It is clear from eq 6-11 that each area (or initial slope) is given as the product of the weight-average electric parameter $\langle r\rangle_{w}$ and the differently averaged relaxation times (or rotary diffusion coefficients). Therefore, if $\langle r\rangle_{w}$ is known, both $\langle\tau\rangle_{z+1}$ and $\langle\tau\rangle_{z}$ may be evaluated separately from any two areas, or $\langle r\rangle_{w}$ from known $\langle\tau\rangle_{z+1}$ and $\langle\tau\rangle_{z}$, provided that the field-off and field-on electric and hydrodynamic properties remain unchanged by the orienting electric field. Under this condition, the relationship shown by expression (1), (2), or (3) should hold. It should be noted that area $A_{\mathrm{R}}$ is related to $\langle\tau\rangle_{z+1}$ alone (the field-on state), but both area $A_{\mathrm{B}}$ and area $A_{\mathrm{D}}$ contain $\langle\tau\rangle_{z+1}$ and $\langle\tau\rangle_{z}$ (the field-on and -off states).

\section{EXPERIMENTAL}

\section{Materials and Measurements}

The fractionated $[\mathrm{Glu}(\mathrm{OBzl})]_{n}$ sample with a weight-average molecular weight of $1.71 \times$ $10^{5}$ and the solvents (2-chloroethanol (CEL), cyclohexanone $(\mathrm{CHN})$, pyridine $(\mathrm{PYN})$, and chloroform (CFM)) were all described previously. ${ }^{15}$ The measurement of signals was carried out at $535 \mathrm{~nm}$ and at $20^{\circ} \mathrm{C}$. The instrumental time constant was $c a .0 .7 \mu$ s for RPEB signals. The apparatus, procedure for measurement, and analysis of the experimental data are given elsewhere. ${ }^{15}$ The contribution of the solvent birefringence to the observed signal was subtracted. The concentration of $[\mathrm{Glu}(\mathrm{OBzl})]_{n}$ was expressed with the residue concentration in $\mathrm{mM}\left(=10^{-3} \mathrm{moldm}^{-3}\right)$.

\section{Evaluation of the RPEB Areas}

The areas were evaluated from the normalized RPEB signal, according to the computeraided curve-fitting method with empirical formulas. ${ }^{13}$ In order to check this method, the areas were manually computed, in some cases, from the RPEB signal digitally plotted on a large-size $1 \times 1 \mathrm{~mm}$ section paper. An actual RPEB signal was compared with the smoothed curve in Figure 2.

A question arises as to whether the area or the initial slope is easier to be determined experimentally with high accuracy. The answer depends on the type of an RPEB apparatus and a solvent in use. If the apparatus delivers a reversing pulse with some instrumental time constant but an electric power enough to prevent it from sagging, the area is a more reliable quantity. If the apparatus produces a sharp square pulse at the initial portion but if a wide pulse duration is difficult to be maintained, the initial slope is better to be measured. If the solvent contributes appreciably to the RPEB signal, ${ }^{15}$ the initial slope method is unworkable, as observed in the present case.

\section{RESULTS AND DISCUSSION}

\section{Normalized RPEB Signal Profiles}

Figure 2 shows an actual RPEB signal of $[\mathrm{Glu}(\mathrm{OBzl})]_{n}$ in 2-chloroethanol (upper half). Since the signal of a dilute solution was weak, 25 observed signals were averaged to improve

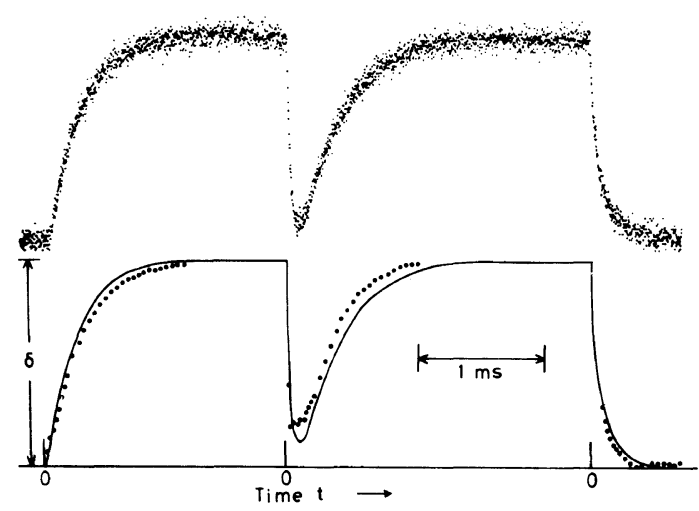

Figure 2. Observed RPEB signal of $[\mathrm{Glu}(\mathrm{OBzl})]_{n}$ in 2-chloroethanol (upper) and a comparison between the smoothed signal profile and computed points (lower). 25 digitized signals were accumulated and averaged. Applied electric field strength: $3.1 \mathrm{kV} \mathrm{cm}^{-1}$. Sampling time: $50 \mathrm{~ns}$ between successive digitized dots (upper). Concentration: $1.12 \mathrm{mM}$. See the text for detail. 
the profile, but this procedure smoothed out the initial slopes to be less reliable. The normalized RPEB profiles were computed from eq 1 , e.g., the buildup protion from observed reverse and decay portions, and so on, as indicated with small points (lower half). If the electric and hydrodynamic properties remain unchanged in the presence and the absence of applied electric fields, the computed points

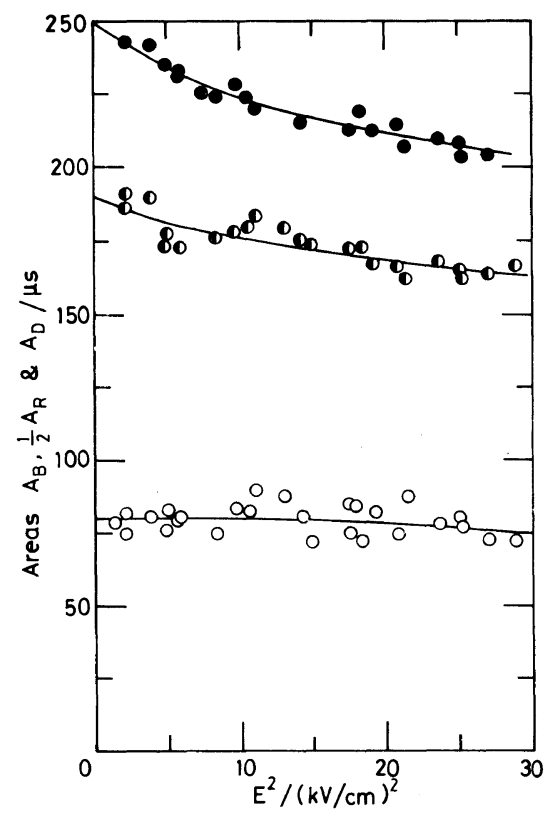

Figure 3. Areas of normalized RPEB signals of [Glu$(\mathrm{OBzl})]_{n}$ in 2-chloroethanol in the low field region. Concentration, $1.12 \mathrm{mM}$; areas in $\mu \mathrm{s}, A_{\mathrm{B}}(\bigcirc) ; \frac{1}{2} A_{\mathrm{R}}(\bigcirc)$; $A_{\text {D }}(\bigcirc)$. should be superimposed upon the observed RPEB signals within an experimental error. Evidently, this is not the case. The result in Figure $2 b$ reveals qualitatively that the electric field affects the properties of $[\mathrm{Glu}(\mathrm{OBzl})]_{n}$ during the buildup process. A comparison should, in principle, be made in the Kerr-law region, i.e., $E^{2} \rightarrow 0$, where eq $1-3$ are strictly applicable, but a single RPEB signal always contains unavoidable random error. Therefore, the area method (eq 3) is the only practical means to estimate the direct field effect on a quantitative basis, considering that the instrumental limitation still remains at present.

\section{Areas of RPEB Signals in the Kerr-Law Region}

Figure 3 shows the areas for buildup, reverse, and decay processes experimentally determined from RPEB signals at low electric fields. Although these area values in each process are scattered due to experimental errors, a large electric field dependence is noticeable for buildup and reverse processes. The area for decay is, on the other hand, nearly independent of field strengths, indicating that the distribution of the chain lengths is narrow for the present $[\mathrm{Glu}(\mathrm{OBzl})]_{n}$ sample. ${ }^{15,33}$ Values of areas were extrapolated to the limiting low field $\left(E^{2} \rightarrow 0\right)$ with appropriate curvatures, which have been shown to depend on the electric parameter $\left\langle\beta^{2}\right\rangle_{w} / 2\langle\gamma\rangle_{w}{ }^{13}$ This parameter can be evaluated from the $\Delta_{m}$ and $t_{m}$ values of the

Table I. Interrelation between areas of RPEB transient signals at the limiting low field for $[\mathrm{Glu}(\mathrm{OBzl})]_{n}$ in helix-forming solvents at $20^{\circ} \mathrm{C}^{\mathrm{a}}$

\begin{tabular}{|c|c|c|c|c|c|c|c|c|}
\hline Solvent & $\frac{\text { Concn. }}{\mathrm{mM}}$ & $\frac{A_{\mathrm{B}}}{(1 / 2) A_{\mathrm{R}}+A_{\mathrm{D}}}$ & $\frac{A_{\mathrm{R}}}{A_{\mathrm{B}}}$ & $\frac{A_{\mathrm{R}}}{A_{\mathrm{D}}}$ & $\frac{A_{\mathrm{B}}}{A_{\mathrm{D}}}$ & $\frac{A_{\mathrm{R}}}{\mu s}$ & $\frac{A_{\mathrm{B}}}{\mu s}$ & $\frac{A_{\mathrm{D}}}{\mu s}$ \\
\hline CEL & 1.12 & $0.93 \pm 0.02$ & $1.5 \pm 0.05$ & $4.7 \pm 0.2$ & $3.1 \pm 0.1$ & $380 \pm 4$ & $250 \pm 5$ & $80 \pm 2$ \\
\hline $\mathrm{CHN}$ & 1.54 & $0.95 \pm 0.02$ & $1.5 \pm 0.1$ & $4.7 \pm 0.2$ & $3.0 \pm 0.2$ & $280 \pm 5$ & $190 \pm 5$ & $60 \pm 5$ \\
\hline PYN & 2.08 & $0.81 \pm 0.02$ & $1.7 \pm 0.1$ & $4.7 \pm 0.2$ & $2.7 \pm 0.2$ & $136 \pm 4$ & $79 \pm 4$ & $29 \pm 2$ \\
\hline CFM & 3.19 & $0.74 \pm 0.04$ & $1.6 \pm 0.1$ & $3.0 \pm 0.2$ & $1.9 \pm 0.2$ & $150 \pm 20$ & $95 \pm 15$ & $50 \pm 5$ \\
\hline CFM & 1.91 & $0.81 \pm 0.02$ & $1.7 \pm 0.1$ & $4.0 \pm 0.2$ & $2.4 \pm 0.2$ & $170 \pm 3$ & $102 \pm 2$ & $42 \pm 2$ \\
\hline
\end{tabular}

a Each value was evaluated by extrapolation of the area or area ratio versus $E^{2}$ plot to zero field $\left(E^{2} \rightarrow 0\right)$; therefore, a small discrepancy always exists between values in the third column and those calculated from the individual $A_{\mathrm{R}} / A_{\mathrm{B}}, A_{\mathrm{R}} / A_{\mathrm{D}}$, and $A_{\mathrm{B}} / A_{\mathrm{D}}$ values, in which experimental errors are large. CEL stands for 2-chloroethanol, $\mathrm{CHN}$ for cyclohexanone, PYN for pyridine, and CFM for chloroform. 
reverse process. ${ }^{5,9,15}$ The extrapolated area values are given in Table $\mathrm{I}$.

\section{Area Relation between Buildup, Reverse, and} Decay Processes

Once individual areas are evaluated in the low field region, the area ratio, $A_{\mathrm{B}} /\left[(1 / 2) A_{\mathrm{R}}+\right.$ $\left.A_{\mathrm{D}}\right]$, can be plotted against $E^{2}$ for detecting any possible field effects on the electric and hydrodynamic properties. Figure 4 shows plots of $A_{\mathrm{B}} /\left[(1 / 2) A_{\mathrm{R}}+A_{\mathrm{D}}\right] v s$. $E^{2}$ for $[\mathrm{Glu}(\mathrm{OBzl})]_{n}$ in four helix-forming solvents. Here again, the dependence of the ratio on applied field strength is noticeable. This dependence differs from one solvent to another, reflecting that values of $\left\langle\beta^{2}\right\rangle_{w} / 2\langle\gamma\rangle_{w}$ are different (not shown) ( $c f$. Figure 4 of ref 13 and Table I of ref 15). Experimental values are very much scattered for the $3.19 \mathrm{mM} \quad[\mathrm{Glu}(\mathrm{OBzl})]_{n}$ solution in chloroform (d), in which molecular aggregation ${ }^{34}$ and the effect of applied electric field on it ${ }^{15}$ have been studied.

In Figure 4, the area ratios extrapolated to the limiting low field are less than unity in four

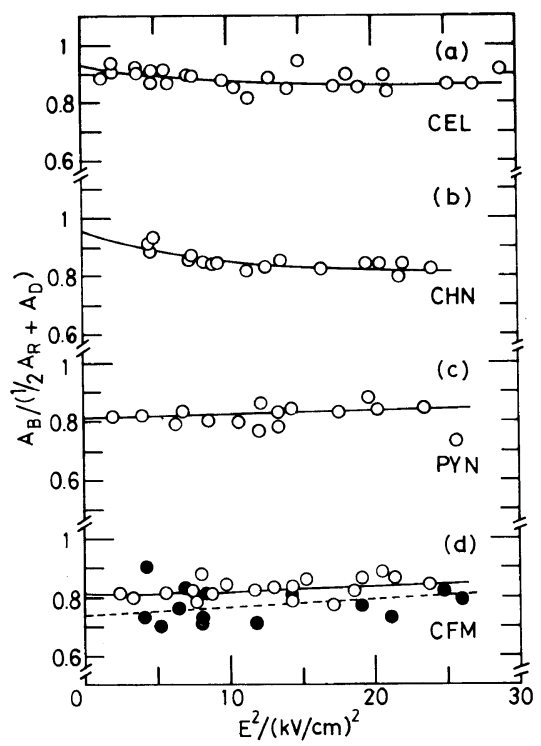

Figure 4. Relationship between areas of RPEB signals for $[\mathrm{Glu}(\mathrm{OBzl})]_{n}$ in helix-forming solvents. Concentrations: (a) $1.12 \mathrm{mM}$ in 2-chloroethanol (CEL); (b) $1.54 \mathrm{mM}$ in cyclohexanone $(\mathrm{CHN})$; (c) $2.08 \mathrm{mM}$ in pyridine $(\mathrm{PYN})$; (d) $3.19 \mathrm{mM}(\mathrm{O})$ and $1.91 \mathrm{mM}(\mathrm{O})$ in chloroform (CFM). solvents, ranging from 0.95 in cyclohexanone to 0.74 in chloroform at a higher concentration. Given in Table I, these values indicate that both, or either, electric and hydrodynamic properties of $[\mathrm{Glu}(\mathrm{OBzl})]_{n}$ in the presence of applied pulse fields differ from those in the absence of the fields; otherwise, the area relationship (eq 2) should hold identically. The direct effect of field on $[\mathrm{Glu}(\mathrm{OBzl})]_{n}$ is, however, only slight in cyclohexanone. In fact, the field-on and field-off conformations of $[\mathrm{Glu}(\mathrm{OBz})]_{n}$ were reported to be the same at more elevated temperatures. ${ }^{13}$ In pyridine and chloroform, area ratios are far less than unity, clearly showing the field-on and field-off properties disagree with each other. The nature of this field effect is not clarified at present. The interaction between solvent and solute molecules may play an important role, e.g., softening of helical structure and easier alignment of polar side-chain groups toward the external field direction.

It becomes evident now that the applied electric field affects the rodlike and nonionized $[\mathrm{Glu}(\mathrm{OBzl})]_{n}$ conformation reversibly even at low field strengths to a varying degree. It is the area method, associated with the RPEB technique, that reveals the so far undetected but important field effect. It will be a future problem to relate, on a quantitative basis, this observed field effect with such factors as the pitch and twist angle of a helical backbone, ${ }^{11}$ helical chain length, ${ }^{13}$ and selective solvation surrounding the helical periphery.

\section{Areas as Electric and Hydrodynamic Parame- ters}

Since the field-on steady-state birefringence $\Delta n$ should be the same in all RPEB processes ( $c f$. eq A10), the ratios of any two of three transient processes are given from eq $6-8$ as

$$
\frac{A_{\mathrm{R}}}{A_{\mathrm{B}}}=\frac{\frac{6\left\langle\beta_{\mathrm{R}}{ }^{2}\right\rangle_{w}}{2\left\langle\gamma_{\mathrm{B}}\right\rangle_{w}} \cdot\left\langle\tau_{\mathrm{R}}\right\rangle_{z+1}}{\frac{4\left\langle\beta_{\mathrm{B}}{ }^{2}\right\rangle_{w}}{2\left\langle\gamma_{\mathrm{B}}\right\rangle_{w}} \cdot\left\langle\tau_{\mathrm{B}}\right\rangle_{z+1}+\left\langle\tau_{\mathrm{B}}\right\rangle_{z}}
$$




$$
\begin{aligned}
& \frac{A_{\mathrm{R}}}{A_{\mathrm{D}}}= \frac{\frac{6\left\langle\dot{\beta}_{\mathrm{R}}{ }^{2}\right\rangle_{w}}{2\left\langle\gamma_{\mathrm{D}}\right\rangle_{w}} \cdot\left\langle\tau_{\mathrm{R}}\right\rangle_{z+1}}{\frac{\left\langle\beta_{\mathrm{D}}{ }^{2}\right\rangle_{w}}{2\left\langle\gamma_{\mathrm{D}}\right\rangle_{w}} \cdot\left\langle\tau_{\mathrm{D}}\right\rangle_{z+1}+\left\langle\tau_{\mathrm{D}}\right\rangle_{z}} \\
& \frac{A_{\mathrm{B}}}{A_{\mathrm{D}}}=\frac{\frac{4\left\langle\beta_{\mathrm{B}}{ }^{2}\right\rangle_{w}}{2\left\langle\gamma_{\mathrm{D}}\right\rangle_{w}} \cdot\left\langle\tau_{\mathrm{B}}\right\rangle_{z+1}+\frac{\left\langle\gamma_{\mathrm{B}}\right\rangle_{w}}{\left\langle\gamma_{\mathrm{D}}\right\rangle_{w}} \cdot\left\langle\tau_{\mathrm{B}}\right\rangle_{z}}{\frac{\left\langle\beta_{\mathrm{D}}{ }^{2}\right\rangle_{w}}{2\left\langle\gamma_{\mathrm{D}}\right\rangle_{w}} \cdot\left\langle\tau_{\mathrm{D}}\right\rangle_{z+1}+\left\langle\tau_{\mathrm{D}}\right\rangle_{z}}
\end{aligned}
$$

These area ratios are expressed as the complex products of electric and hydrodynamic parameters characteristic of the buildup, reverse, and decay processes. If these parameters all remain unaltered by an applied electric field, i.e., no direct field effect is involved, eq 12-14 can then be reduced to simpler forms, the parameters being common to all three processes (i.e., $\left\langle r_{\mathrm{B}}\right\rangle=\left\langle r_{\mathrm{R}}\right\rangle=\left\langle r_{\mathrm{D}}\right\rangle$ and $\left\langle\tau_{\mathrm{B}}\right\rangle=$ $\left.\left\langle\tau_{\mathbf{R}}\right\rangle=\left\langle\tau_{\mathbf{D}}\right\rangle\right)$

$$
\begin{aligned}
\frac{A_{\mathrm{R}}}{A_{\mathrm{B}}}= & \frac{6\langle r\rangle_{w} \cdot \frac{\langle\tau\rangle_{z+1}}{\langle\tau\rangle_{z}}}{4\langle r\rangle_{w} \cdot \frac{\langle\tau\rangle_{z+1}}{\langle\tau\rangle_{z}}+1}=\frac{6 Q}{4 Q+1} \\
\frac{A_{\mathrm{R}}}{A_{\mathrm{D}}} & =\frac{6\langle r\rangle_{w} \cdot \frac{\langle\tau\rangle_{z+1}}{\langle\tau\rangle_{z}}}{\langle r\rangle_{w} \cdot \frac{\langle\tau\rangle_{z+1}}{\langle\tau\rangle_{z}}+1}=\frac{6 Q}{Q+1} \\
\frac{A_{\mathrm{B}}}{A_{\mathrm{D}}}= & \frac{\left\langle\langle r\rangle_{w} \cdot \frac{\langle\tau\rangle_{z+1}}{\langle\tau\rangle_{z}}+1\right.}{\langle r\rangle_{w} \cdot \frac{\langle\tau\rangle_{z+1}}{\langle\tau\rangle_{z}}+1}=\frac{4 Q+1}{Q+1}
\end{aligned}
$$

where $Q=\langle r\rangle_{w}\langle\tau\rangle_{z+1} /\langle\tau\rangle_{z}$, the electro-hydrodynamic parameter. Equations 15-17 show that these area ratios contain the product of the electric and hydrodynamic quantities, which are again unseparable. These area ratios vary continuously between two limits, whereas $A_{\mathrm{B}} /\left[(1 / 2) A_{\mathrm{R}}+A_{\mathrm{D}}\right]$ remains unity (cf. eq 2 ) independent of $Q$, as shown below.

\begin{tabular}{lclll}
\hline$Q$ & $A_{\mathrm{B}} /\left(\frac{1}{2} A_{\mathrm{R}}+A_{\mathrm{D}}\right)$ & $A_{\mathrm{R}} / A_{\mathrm{B}}$ & $A_{\mathrm{R}} / A_{\mathrm{D}}$ & $A_{\mathrm{B}} / A_{\mathrm{D}}$ \\
\hline 0 & 1 & 0 & 0 & 1 \\
$\infty$ & 1 & 1.5 & 6.0 & 4.0 \\
\hline
\end{tabular}

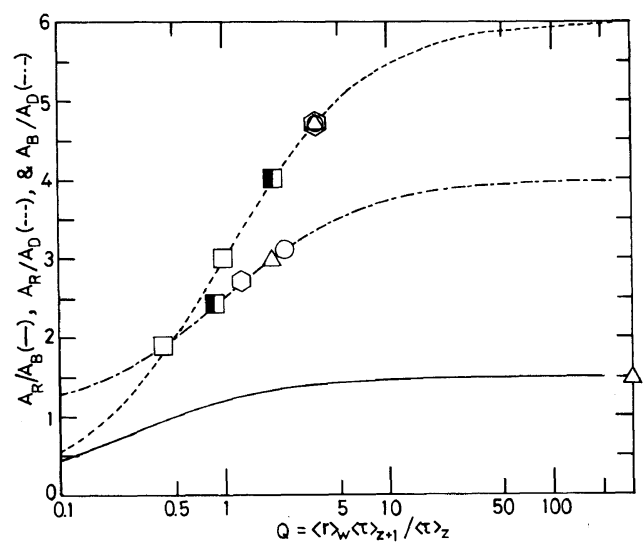

Figure 5. Theoretical curves of three different area ratios against the electro-hydrodynamic parameter $Q . A_{\mathrm{R}} / A_{\mathrm{B}}$, $(\longrightarrow) ; A_{\mathrm{R}} / A_{\mathrm{D}},(---) ; A_{\mathrm{B}} / A_{\mathrm{D}},(-\cdot-\cdot-)$. Symbols are experimental values: $(O)$ in 2-chloroethanol; $(\triangle)$ in cyclohexanone; $(O)$ in pyridine; $(\square, 3.19 \mathrm{mM}$; $\square$, $1.91 \mathrm{mM}$ ) in chloroform.

Figure 5 shows the plot of eq 15-17 against $Q$ on a semilogarithmic scale. Area ratios $\left(A_{\mathrm{R}} / A_{\mathrm{B}}, A_{\mathrm{R}} / A_{\mathrm{D}}\right.$, and $\left.A_{\mathrm{B}} / A_{\mathrm{D}}\right)$ tend to approach the high limiting values rapidly $(Q \simeq 10)$, the largest change occurring for $A_{\mathrm{R}} / A_{\mathrm{D}}(0-6.0)$. In Figure 5 the experimental values in Table I are also shown with different symbols. If no direct field effect is present in the transient processes, three experimental area ratios $A_{\mathrm{R}} / A_{\mathrm{B}}, A_{\mathrm{R}} / A_{\mathrm{D}}$, and $A_{\mathrm{B}} / A_{\mathrm{D}}$ of a $[\mathrm{Glu}(\mathrm{OBzl})]_{n}$ solution should yield the same $Q$ value on the abscissa. Apparently this is not the case in each solvent. Both $A_{\mathrm{R}} / A_{\mathrm{D}}$ and $A_{\mathrm{B}} / A_{\mathrm{D}}$ seem to yield $Q$ values lower than they should, on the basis of $\left\langle\beta^{2}\right\rangle_{w} / 2\langle\gamma\rangle_{w}$ values which were found to be much larger in a previous study. ${ }^{15}$ Probably except for cyclohexanone, $A_{\mathrm{R}} / A_{\mathrm{B}}$ values exceed the theoretical limit of 1.5 at infinitely large $Q$, which corresponds to the pure permanent dipole orientation $\left(\langle r\rangle_{w}=\left\langle\beta^{2}\right\rangle_{w} / 2\langle\gamma\rangle_{w}=\infty\right)$. 
Any values greater than 1.5 , e.g., 1.7 in pyridine, should be interpreted as being due to the direct field effect on the electric and hydrodynamic properties of $[\mathrm{Glu}(\mathrm{OBzl})]_{n}$.

The above results altogether indicate that the field-on reverse process yields an $A_{\mathrm{R}}$ larger than it should be expected. The data in Figure 5 may be interpreted, on a qualitative basis, as being that the electric moments $(\mu$ and $\Delta \alpha E$ ) are increased by the applied electric field, if the chain length of the $[\mathrm{Glu}(\mathrm{OBzl})]_{n}$ helix remains unchanged in the transient processes. ${ }^{13}$ Any quantitative discussions are difficult to be made on the change in the electric moment and the hydrodynamic chain length in the presence of applied field, since such a change may be an interplay of many variables $(\mu, \Delta \alpha$, and $\tau)$ involved in the parameter $Q$. The change of electro-hydrodynamic parameters should be time-dependent; for example, the change of $\mu$ may be either instantaneous or gradual, varying with the pulse duration. ${ }^{13}$ Needles to say, such a change is reversible and the field-off state is always recovered upon removal of an external field. Any further discussions are beyond the scope of the present work.

\section{RPEB vs. SPEB Measurements}

From conventional SPEB measurements at weak fields, the areas are determined only for buildup and decay signals. The area ratio for a polydisperse polymer sample is given by eq 14 or 17. If a polymer system is monodisperse regarding chain length, the electro-hydrodynamic parameter $Q$ reduces to $r \equiv \beta(l)^{2} / 2 \gamma(l)$ $\left(\langle r\rangle_{w}=r(l)=r\right.$ and $\left.\langle\tau\rangle_{z}=\langle\tau\rangle_{z+1}\right)$. Equation 17 reduces to

$$
\frac{A_{\mathrm{B}}}{A_{\mathrm{D}}}=\frac{4 r+1}{r+1}
$$

This expression was first proposed by Yoshioka and Watanabe, ${ }^{35}$ who suggested that the ratio is 4 , as $r \rightarrow \infty$ (the permanent dipole moment orientation), whereas it is 0 , as $r \rightarrow 0$ (the electronic induced moment orientation). The notion that the $r$ value can be evaluated from eq 18 is correct only for the monodisperse polymer system, in contrast with the original ${ }^{35}$ and subsequent reports. ${ }^{36-38}$ It should be noted that the ratio $A_{\mathrm{B}} / A_{\mathrm{D}}$ alone cannot reveal the possible effects of applied pulse field on the polymer conformation.

As already presented in detail in the preceding sections, an RPEB measurement at weak fields makes it possible to detect not only reversible conformational transition or change in the electric and hydrodynamic properties but also its existence and likely causes. This is because two additional ratios $A_{\mathrm{R}} / A_{\mathrm{B}}$ and $A_{\mathrm{R}} / A_{\mathrm{D}}$ are available, together with the area relationship (eq 2). Hence, the advantage of the RPEB over the SPEB measurement is evident.

\section{Area vs. Initial Slope in the Kerr-Law Region}

The initial slopes for buildup, reverse, and decay processes ( $c f$. Figure 1) are related to each other by the identity (eq 3), which is close to the relationship between areas (eq 2). Nishinari and Yoshioka showed in the SPEB study that the initial slope in the buildup process is expressed as the product of the electric and hydrodynamic parameters for a monodisperse system. ${ }^{39,40)}$ Although the initial slopes in the Kerr-law region contain a large experimental error, the combination of areas with initial slopes in three transient processes yields potentially very useful expressions, in which the electric parameters are eliminated. From eq 7 and 10, the field-on reverse process of an RPEB signal yields the relaxation time and the rotary diffusion constant $(\Theta=1 / 6 \tau)$ :

$$
\frac{A_{\mathrm{R}}}{S_{\mathrm{R}}}=-\frac{3\langle\tau\rangle_{z+1}}{\langle 1 / \tau\rangle_{z+1}}
$$

From eq 6,8 , and 9 , the following expressions are derived for the field-on and field-off processes:

$$
\frac{4 A_{\mathrm{D}}-A_{\mathrm{B}}}{S_{\mathrm{B}}}=\frac{3 A_{\mathrm{B}}-2 A_{\mathrm{R}}}{S_{\mathrm{B}}}=\frac{3\langle\tau\rangle_{z}}{\langle 1 / \tau\rangle_{z}}
$$




$$
\frac{A_{\mathrm{B}}-A_{\mathrm{D}}}{S_{\mathrm{B}}+S_{\mathrm{D}}}=-\frac{3\langle\tau\rangle_{z+1}}{\langle 1 / \tau\rangle_{z+1}}
$$

It should be noted that eq $19-21$ contain only hydrodynamic parameters.

Various averages of the relaxation time $\langle\tau\rangle$ and the rotary diffusion constant $\langle 1 / 6 \tau\rangle$ can be calculated theoretically from the distribution function of chain length, $l f_{n}(l)$, and the appropriate hydrodynamic equation for rodlike molecules. ${ }^{5,15,40-42}$ The function $l f_{n}(l)$ may also be estimated experimentally by gel permeation chromatography for helical polymers, for which the length is proportional to molecular weight. Thus, the effect of applied electric pulse on the reversible chain elongation or contraction, as predicted in previous papers, ${ }^{11,13}$ can be detected with the aid of eq 19-21. In order to take a full advantage of the initial slope method, a technical innovation for a fast and well-defined square-wave pulse generator is eagerly awaited. This is our future goal. Finally, the RPEB technique should be highly recommended for obtaining detailed information on the structural properties of polymers, as fully elaborated in this and previous studies. ${ }^{1-17}$

\section{CONCLUSIONS}

In the course of field orientation of $[\mathrm{Glu}(\mathrm{OBzl})]_{n}$ helices, the electric and hydrodynamic properties in some organic solvents must be modified to a varying degree by an applied electric pulse, even if such measurements are carried out in the Kerr-law region. For detecting these changes that occur reversibly by application of pulse fields, analysis of transient RPEB signals is essential; in particular, the area method is highly recommendable. In the limiting low field region, a universal relation, $A_{\mathrm{B}}=(1 / 2) A_{\mathrm{R}}+A_{\mathrm{D}}$, should hold, if no direct field effect is present.

The steady-state birefringence has been studied in the past under diverse conditions. From the field strength dependence, a great deal of information has been accumulated on the electric and optical properties of polymers including rodlike helices. In a strict sense, however, these field-on properties do not necessarily represent the electrooptical and hydrodynamic properties of a given polymer system in the absence of applied fields, unless the area relationship must be confirmed in advance. This point has long been overlooked but clarified in the present work for the first time.

\section{APPENDIX}

Theoretical RPEB expressions are summarized below for the normalized signal of a polydisperse polymer system in the Kerr-law region.

\section{[I] Buildup Process}

(1) Normalized birefringence $\Delta_{\mathrm{B}}(t)$ :

$$
\Delta_{\mathrm{B}}(t) \equiv \frac{\Delta n_{\mathrm{B}}(t)}{\Delta n_{\mathrm{B}}(\infty)}=1-\frac{\int_{0}^{\infty} \Delta n_{\mathrm{B}}(\infty, l)\left\{\frac{3 \beta(l)^{2} \mathrm{e}^{-2 \Theta_{11}(l) t}-\left[\beta(l)^{2}-4 \gamma(l)\right] \mathrm{e}^{-6 \Theta_{11}(l) t}}{2\left[\beta(l)^{2}+2 \gamma(l)\right]}\right\} \mathrm{d} l}{\int_{0}^{\infty} \Delta n_{\mathrm{B}}(\infty, l) \mathrm{d} l}
$$

(2) Area $A_{\mathrm{B}}$ :

$$
A_{\mathrm{B}}=\int_{0}^{\infty}\left[1-\Delta_{\mathrm{B}}(t)\right] \mathrm{d} t=\frac{\int_{0}^{\infty} \Delta n_{\mathrm{B}}(\infty, l) \tau(l)\left[1+\frac{3 \beta(l)^{2}}{\beta(l)^{2}+2 \gamma(l)}\right] \mathrm{d} l}{\int_{0}^{\infty} \Delta n_{\mathrm{B}}(\infty, l) \mathrm{d} l}
$$


(3) Initial slope $S_{\mathrm{B}}$ :

$$
S_{\mathrm{B}} \equiv\left(\frac{\mathrm{d} \Delta_{\mathrm{B}}}{\mathrm{d} t}\right)_{t \rightarrow 0}=\frac{\int_{0}^{\infty} \Delta n_{\mathrm{B}}(\infty, l)\left[\frac{2 \tau^{-1}(l) \gamma(l)}{\beta(l)^{2}+2 \gamma(l)}\right] \mathrm{d} l}{\int_{0}^{\infty} \Delta n_{\mathrm{B}}(\infty, l) \mathrm{d} l}
$$

\section{[II] Reverse Process}

(1) Normalized birefringence $\Delta_{\mathrm{R}}(t)$ :

$$
\Delta_{\mathbf{R}}(t) \equiv \frac{\Delta n_{\mathrm{R}}(t)}{\Delta n_{\mathrm{R}}(\infty)}=1-\frac{\int_{0}^{\infty} \Delta n_{\mathrm{R}}(\infty, l)\left[\frac{3 \beta(l)^{2}}{\beta(l)^{2}+2 \gamma(l)}\right]\left(\mathrm{e}^{-2 \Theta_{11}(l) t}-\mathrm{e}^{-6 \Theta_{11}(l) t}\right) \mathrm{d} l}{\int_{0}^{\infty} \Delta n_{\mathrm{R}}(\infty, l) \mathrm{d} l}
$$

(2) Area $A_{\mathrm{R}}$ :

$$
A_{\mathrm{R}}=\int_{0}^{\infty}\left[1-\Delta_{\mathrm{R}}(t)\right] \mathrm{d} t=\frac{\int_{0}^{\infty} \Delta n_{\mathrm{R}}(\infty, l) \tau(l)\left[\frac{6 \beta(l)^{2}}{\beta(l)^{2}+2 \gamma(l)}\right] \mathrm{d} l}{\int_{0}^{\infty} \Delta n_{\mathrm{R}}(\infty, l) \mathrm{d} l}
$$

(3) Initial slope $S_{\mathbf{R}}$ :

$$
S_{\mathrm{R}} \equiv\left(\frac{\mathrm{d} \Delta_{\mathrm{R}}}{\mathrm{d} t}\right)_{t \rightarrow 0}=-\frac{\int_{0}^{\infty} \Delta n_{\mathrm{R}}(\infty, l)\left[\frac{2 \beta(l)^{2} \tau(l)^{-1}}{\beta(l)^{2}+2 \gamma(l)}\right] \mathrm{d} l}{\int_{0}^{\infty} \Delta n_{\mathrm{R}}(\infty, l) \mathrm{d} l}
$$

\section{[III] Decay Process}

(1) Normalized birefringence $\Delta_{\mathrm{D}}(t)$ :

$$
\Delta_{\mathrm{D}}(t) \equiv \frac{\Delta n_{\mathrm{D}}(t)}{\Delta n_{\mathrm{D}}(0)}=\frac{\int_{0}^{\infty} \Delta n_{\mathrm{D}}(0, l) \mathrm{e}^{-6 \Theta_{11}(l) t} \mathrm{~d} l}{\int_{0}^{\omega} \Delta n_{\mathrm{D}}(0, l) \mathrm{d} l}
$$

(2) Area $A_{\mathrm{D}}$ :

$$
A_{\mathrm{D}}=\int_{0}^{\infty} \Delta n_{\mathrm{D}}(t) \mathrm{d} t=\frac{\int_{0}^{\infty} \Delta n_{\mathrm{D}}(0, l) \tau(l) \mathrm{d} l}{\int_{0}^{\infty} \Delta n_{\mathrm{D}}(0, l) \mathrm{d} l}
$$

(3) Initial slope $S_{\mathrm{D}}$ : 


$$
S_{\mathrm{D}} \equiv\left(\frac{\mathrm{d} \Delta_{\mathrm{D}}}{\mathrm{d} t}\right)_{t \rightarrow 0}=-\frac{\int_{0}^{\infty} \Delta n_{\mathrm{D}}(0, l) \tau(l)^{-1} \mathrm{~d} l}{\int_{0}^{\infty} \Delta n_{\mathrm{D}}(0, l) \mathrm{d} l}
$$

Notations are $\beta(l)=\mu(l) E / k T, \gamma(l)=\Delta \alpha(l) E^{2} /$ $2 k T$, where $E$ is the applied electric field strength, $k$ is the Boltzmann constant, and $T$ is the absolute temperature. $\tau(l)$ and $\Theta_{11}(l)$ are the rotational relaxation time and the rotary diffusion coefficient, respectively, of the whole molecule around the transverse axis, i.e., $6 \Theta_{11}(l)=1 / \tau(l)$. The steady-state birefringence in the buildup process $\Delta n_{\mathrm{B}}(\infty)$ is given as $\left(\Delta n_{\mathrm{B}}(\infty)=\Delta n_{\mathrm{R}}(\infty)=\Delta n_{\mathrm{D}}(0)\right)$

$$
\begin{aligned}
& \Delta n_{\mathrm{B}}(\infty)=\int_{0}^{\infty} \Delta n_{\mathrm{B}}(\infty, l) \mathrm{d} l \\
& \quad=\left(\frac{2 \pi C_{v}}{15}\right)\left(\frac{\Delta g}{n}\right) \int_{0}^{\infty} \phi(l)\left[\beta(l)^{2}+2 \gamma(l)\right] \mathrm{d} l
\end{aligned}
$$

where $C_{v}$ is the mean volume fraction of solutes in a polydisperse system and $(\Delta g / n)$ is the reduced optical anisotropy factor, $n$ being the refractive index of solution, and $\phi(l)$ is the fraction of solutes with the length $l .(\Delta g / n)$ is generally assumed to be independent of the chain length for a thin, rodlike molecule. ${ }^{19,43}$

\section{REFERENCES}

1. K. Yamaoka and S. Yamamoto, Polym. Prepr. Jpn., 34, 1014 (1985). For Part IV of this series, see ref 15.

2. K. Yamaoka and K. Ueda, J. Phys. Chem., 84, 1422 (1980).

3. K. Yamaoka, T. Ichibakase, K. Ueda, and K. Matsuda, J. Am. Chem. Soc., 102, 5109 (1980).

4. K. Yamaoka and K. Matsuda, Macromolecules, 13, 1558 (1980)

5. K. Yamaoka and K. Ueda, J. Phys. Chem., 86, 406 (1982).

6. K. Yoshioka, M. Fujimori, K. Yamaoka, and K. Ueda, Int. J. Biol. Macromol., 4, 55 (1982).

7. K. Yamaoka and K. Ueda, Chem. Lett., 545 (1983).

8. K. Matsuda, J. Sci. Hiroshima Univ. Ser. A, 47, 41 (1983).

9. K. Ueda, M. Nomura, and K. Yamaoka, Bio- polymers, 22, 2077 (1983).

10. K. Ueda, M. Mimura, and K. Yamaoka, Biopolymers, 23, 1667 (1984).

11. K. Ueda, Bull. Chem. Soc. Jpn., 57, 2703 (1984).

12. K. Yamaoka and K. Matsuda, J. Phys. Chem., 89, 2779 (1985).

13. K. Yamaoka, S. Yamamoto, and K. Ueda, J. Phys. Chem., 89, 5192 (1985).

14. K. Yamaoka, S. Yamamoto, and K. Ueda, Biopolymers, 26, 673 (1987).

15. K. Yamaoka, S. Yamamoto, and I. Kosako, Polym. J., 19, 951 (1987).

16. K. Yamaoka, I. Kosako, and K. Gekko, Polym. J., 21, 107 (1989)

17. I. Tinoco, Jr. and K. Yamaoka, J. Phys. Chem., 63, 423 (1959).

18. M. Matsumoto, H. Watanabe, and K. Yoshioka, Biopolymers, 6, 929 (1968).

19. M. Matsumoto, H. Watanabe, and K. Yoshioka, Biopolymers, 9, 1307 (1970).

20. M. Matsumoto, H. Watanabe, and K. Yoshioka, Biopolymers, 11, 1711 (1972).

21. M. Matsumoto, H. Watanabe, and K. Yoshioka, Biopolymers, 121729 (1973).

22. C. T. O'Konski, K. Yoshioka, and W. H. Orttung, J. Phys. Chem., 63, 1558 (1959).

23. K. Kikuchi and K. Yoshioka, Biopolymers, 12, 2667 (1973).

24. K. Kikuchi and K. Yoshioka, Biopolymers, 15, 1669 (1976).

25. M. Fujimori, K. Kikuchi, K. Yoshioka, and S. Kubota, Biopolymers, 18, 2005 (1979).

26. K. Yoshioka, H. Watanabe, K. Kikuchi, and M. Fujimori, Sci. Pap. Coll. Gen. Educ. Univ. Tokyo, 29, 139 (1979).

27. K. Yoshioka, K. Kikuchi, and M. Fujimori, Biophys. Chem., 11, 369 (1980).

28. K. Yoshioka, M. Fujimori, and K. Kikuchi, Int. J. Biol. Macromol., 2, 213 (1980).

29. H. Watanabe and K. Yoshioka, "Electro-optics and Dielectrics of Macromolecules and Colloids," B. R. Jennings, Ed., Plenum Press, New York, N. Y., 1979, pp. $345-352$.

30. M. Matsumoto, H. Watanabe, and K. Yoshioka, $J$. Phys. Chem., 74, 2182 (1970).

31. H. Watanabe and A. Morita, J. Chem. Phys., 73, 5884 (1980).

32. H. Watanabe and A. Morita, Adv. Chem. Phys., 56, 255 (1984).

33. K. Yamaoka and K. Fukudome, Bull. Chem. Soc. Jpn., 56, 60 (1983).

34. R. Sakamoto and M. Watanabe, "Contemporary Topics in Polymer Science," Vol. 4, W. J. Bailey and T. Tsuruta, Ed., Plenum Press, New York, N.Y., 1984, pp 259-268.

35. K. Yoshioka and H. Watanabe, Nippon Kagaku Zasshi, 84, 626 (1963). 
36. E. Fredericq and C. Houssier, "Electric Dichroism and Electric Birefringence," Clarendon Press, Oxford, 1973, pp 48-53.

37. C. Marion, B. Roux, and J-C. Bernengo, Makromol. Chem., 187, 1647 (1984).

38. G. Mithieux, F. Chauvin, B. Roux, and B. Rousset, Biophys. Chem., 22, 307 (1985).

39. K. Nishinari and K. Yoshioka, Kolloid-Z. Z. Polym., 235, 1189 (1969).

40. K. Nishinari and K. Yoshioka, Kolloid-Z. Z. Polym.,
240, 831 (1970).

41. K. Matsuda and K. Yamaoka, Bull. Chem. Soc. Jpn., 55, 1727 (1982).

42. K. Ueda and K. Yamaoka, Bull. Chem. Soc. Jpn., 56, 2390 (1983).

43. A. Peterlin and H. A. Stuart, "Hand- und Jahrbuch der Chemischen Physik," Bd. 8, von A. Eucken and K. L. Wolf, Ed., Abt. 1B, Akad, Verlagsges., Leipzig, 1943, pp $1-115$. 